\title{
Validation of Analytic Methods for Combining Evidence Sources in Biosurveillance
}

\author{
Howard Burkom*, Yevgeniy Elbert, Liane Ramac-Thomas and Christopher Cuellar \\ Johns Hopkins Applied Physics Laboratory, Laurel, MD, USA
}

\section{Objective}

This presentation aims to reduce the gap between multivariate analytic surveillance tools and public health acceptance and utility. We developed procedures to verify, calibrate, and validate an evidence fusion capability based on a combination of clinical and syndromic indicators and limited knowledge of historical outbreak events.

\section{Introduction}

Recent years' informatics advances have increased availability of various sources of health-monitoring information to agencies responsible for disease surveillance. These sources differ in clinical relevance and reliability, and range from streaming statistical indicator evidence to outbreak reports. Information-gathering advances have outpaced the capability to combine the disparate evidence for routine decision support. In view of the need for analytical tools to manage an increasingly complex data environment, a fusion module based on Bayesian networks (BN) was developed in 2011 for the Dept. of Defense (DoD) Electronic Surveillance System for the Early Notification of Community-Based Epidemics (ESSENCE). In 2012 this module was expanded with syndromic queries, data-sensitive algorithm selection, and hierarchical fusion network training [1]. Subsequent efforts have produced a full fusion-enabled version of ESSENCE for beta testing, further upgrades, and a software specification for live DoD integration. Beta test reviewers cited the reduced alert burden and the detailed evidence underlying each alert. However, only 39 reported historical events were available for training and calibration of 3 networks designed for fusion of influenza-like-illness, gastrointestinal, and fever syndrome categories. The current presentation describes advances to formalize the network training, calibrate the component alerting algorithms and decision nodes together for each $\mathrm{BN}$, and implement a validation strategy aimed at both the ESSENCE public health user and machine learning communities.

\section{Methods}

Hierarchical $\mathrm{BN}$ training was automated with an optimization method to seek conditional probability table (CPT) entries for each subnetwork that would produce desired results from given combinations of algorithm output states. For example, a combination of red alert for laboratory test orders, multiple recent yellow alerts for filled prescriptions, and multiple recent red alerts for ICD-based visits would produce a level of concern above $90 \%$ for outbreak investigation using the network, based on results of algorithms applied to the syndromic data streams for each evidence type. The derived CPTs minimize the difference between the desired and computed BN outputs for all state combinations.

For validation in the absence of sufficient reported events, we searched algorithm results from all selected data streams in 3.75 years of data from 289 military facilities to derive $\sim 100$ historical events for each fusion type. We adopted a cross-validation strategy by partitioning these events into 10 sets, deriving the optimal set of BN and algorithm thresholds with each $10 \%$ removed, and applying these thresholds to the remaining $10 \%$. Each optimal threshold combination maximized the median odds ratio of the BN outbreak decision node, for alerting during outbreak vs non-outbreak days, over all training events. A single set of thresholds was then used for external validation using the 39 reported events.

\section{Results}

Within each fusion type, the cross-validation strategy produced consistent combinations of algorithm and $\mathrm{BN}$ decision thresholds, and the resultant overall threshold choices yielded $88 \%$ sensitivity to reported events, a $10-15 \%$ improvement over the original demonstration module.

\section{Conclusions}

We implemented training and validation methods combining methods of the disease surveillance and machine learning communities. The automated BN methods yielded an increase in sensitivity over previous heuristic methods, and the cross-validation supported the $\mathrm{BN}$ strategy for prospective use in ESSENCE. Increased epidemiologist input is needed for more granular, effective use of data and community acceptance. Extension to other surveillance environments (e.g. civilian state or province) will require expert involvement regarding both available data and the objectives/constraints of those environments.

\section{Keywords}

Bayesian Network; validation; multivariate; ESSENCE

\section{References}

Burkom H, Elbert Y, Ramac-Thomas L, Cuellar C, Hung V, Refinement of a Population-Based Bayesian Network for Fusion of Health Surveillance Data, (2013) Online Journal of Public Health Informatics, 5(1), DOI: http://dx.doi.org/10.5210\%2Fojphi.v5i1.4413

\author{
*Howard Burkom \\ E-mail: howard.burkom@jhuapl.edu
}

